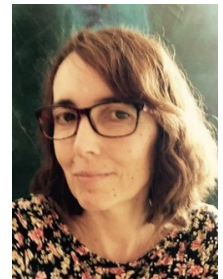

\title{
La integración de los dispositivos móviles en el entorno de trabajo de los profesionales de la traducción y la interpretación
}

Rocío del Pozo Caamaño Traductora técnica e intérprete médico (autónoma)

\section{RESUMEN}

En este artículo se hace un repaso por los dispositivos móviles que han supuesto una cierta revolución en la forma de trabajar de traductores e intérpretes. En poco espacio podemos llevar mucha información, documentarnos para un futuro trabajo y atender a nuestros clientes desde cualquier sitio. El único requisito es tener conexión y un dispositivo móvil.

Palabras clave: dispositivo móvil, tableta, bolígrafo digital, aplicación, interpretación, traducción, teléfono móvil, reloj inteligente

\section{ABSTRACT}

This article is a review of mobile devices that have brought about a revolution in the way translators and interpreters work. In very little space, we can now carry a lot of information, document ourselves for future jobs and answer our clients from anywhere. The only requirement is to have a connection and a smart mobile device.

Keywords: smart mobile device, tablet, stylus, app, interpreting, translation, smart phone, smart watch

\section{RESUM}

En aquest article es fa un repàs dels dispositius mòbils, que han suposat una certa revolució en la manera de treballar de traductors i d'intèrprets. En poc espai podem portar molta informació, documentar-nos per a un proper treball $i$ atendre els nostres clients des de qualsevol lloc. L'únic requisit és tenir connexió i un dispositiu mòbil.

Paraules clau: dispositiu mòbil, tauleta, bolígraf digital, aplicació, interpretació, traducció, telèfon mòbil, rellotge intel-ligent

\section{Introducción}

La tecnología ha venido para quedarse, como se suele decir. $Y$ en el mundo de la traducción y de la interpretación la tecnología móvil en concreto ha supuesto un salto cualitativo importante a varios niveles. Por un lado, nos ofrece oportunidades para aprender, desarrollar nuestras especialidades y mejorar nuestros conocimientos lingüísticos fácilmente desde cualquier sitio y de forma gratuita en muchos casos. Por otro lado, nos permite estar conectados en todo momento, incluso durante esos periodos de tiempo muerto que se producen por ejemplo si tenemos que hacer desplazamientos $u$ otras actividades que nos alejan de nuestra oficina y que en la época pre-tecnología móvil implicaban una interrupción en nuestro trabajo como traductor.

Ya no necesitamos estar delante de nuestro ordenador, en nuestra oficina. Solo necesitamos un teléfono móvil inteligente, un phablet (un híbrido entre una tableta y un teléfono móvil), una tableta o un incluso un reloj inteligente para tener toda la información que deseemos, o que necesitemos, a nuestro alcance al instante. $Y$ no se trata solo de acceso a la información, sino también de las aplicaciones que existen para ayudarnos en las distintas 
tareas de traducción e interpretación, como son la toma de notas en interpretación y la organización de la información.

\section{Dispositivos móviles para la toma de notas}

Como el mundo del intérprete ya es móvil de por sí, la tecnología móvil nos ha traído una manejabilidad que antes no existía incluso en áreas como la toma de notas, que podemos hacer directamente en los dispositivos móviles.

La toma de notas, un aspecto clave en interpretación consecutiva, se ha hecho tradicionalmente en un cuaderno. Actualmente contamos con la posibilidad de tomar notas directamente en las tabletas mediante el uso de un bolígrafo digital, stylus, apropiado a cada tipo de tableta y una aplicación de toma de notas. El mercado ofrece distintos stylus, a una variedad de precios, que ofrecen precisión y fiabilidad para un intérprete de consecutiva. Asimismo, hay varias aplicaciones para la toma de notas, tanto para iOS como para Android, y en muchos casos gratuitas. Entre algunas de esas aplicaciones de escritura a mano se encuentran:

- Penultimate de Evernote, gratuita, para iOS, que ofrece varias opciones como color, grosor del trazo, forma del papel, postura horizontal o vertical del papel, se puede escribir con el dedo o con un stylus.

- MyScript Memo, un poco más básico, gratuito, para iOS, con posibilidades de añadir funciones.

- Notability es otra aplicación para iOS, no gratuita pero asequible, que incluye la posibilidad de grabar el audio al mismo tiempo. Además, el audio grabado se puede sincronizar con las notas. Sin embargo, esta función de grabación del audio podría no estar permitida en ciertas áreas como es el caso de la interpretación médica consecutiva que se realiza en hospitales ya que la sesión de interpretación está supeditada al acuerdo de confidencialidad.

¿Qué ventajas nos trae esto? Al trabajar directamente en la pantalla de nuestra tableta podemos compartir la pantalla con nuestro glosario terminológico personal, o cualquier fuente de referencia que nos hayamos preparado para la sesión de interpretación. Además, hay menos riesgos de que nos olvidemos el cuaderno de notas, o que traspapelemos algunas notas, y de que pongamos en peligro la confidencialidad de la información. En cambio, pocas veces se nos olvidaría la tableta o bien si eso ocurriera no se podría acceder a la información fácilmente gracias a la contraseña. A lo que podemos añadir que es muy sencillo borrar las notas sin dejar rastro y sin ningún tipo de riesgo.

Si usamos la tableta para tomar notas, el hecho de que pesan poco y ocupan poco espacio es esencial. Para un intérprete médico, por ejemplo, que en muchas ocasiones debe interpretar de pie es una ventaja indudable. Y, sin embargo, todo lo que necesitaría cabe ahí, en la tableta, como dice Holly Behl, intérprete jurídica, en una entrevista que le hizo Aida González su blog: «[...] ahora tengo el material de referencia, el bolígrafo, el cuaderno, la lista de tareas pendientes y la lectura para el trayecto todo junto en un único aparato que apenas pesa o abulta» (en Aventuras de una traductora-intérprete en Madrid).

\section{Dispositivos móviles y glosarios terminológicos}

Una de las ventajas claras es que los traductores e intérpretes pueden llevar todas las fuentes de referencia que necesiten en el dispositivo móvil que usen, entre ellas aplicaciones de diccionarios o para crear sus propios glosarios, pueden acceder a diccionarios en línea, sincronizar sus datos, tener aplicaciones para gestionar el tiempo y los archivos, y otros recursos lingüísticos. 
Hay aplicaciones para crear glosarios terminológicos multilingües propios y todas ellas permiten además importar otros glosarios y compartirlos, entre otras funciones. Se pueden destacar las siguientes recomendadas en varios blogs de traductores e intérpretes profesionales (Drechsel, 2016; Drechsel y Goldsmith, 2016; Goldsmith, 2015; González Álamo, 2016; Rioja, 2014):

- Tap Forms es una base de datos para iOS con innumerables usos que se puede editar para crear un glosario multilingüe. Se puede sincronizar fácilmente mediante iCloud o Dropbox.

- Memento Database es una base de datos para Android que también se puede usar para crear glosarios propios. Se puede sincronizar con Google Sheets, y desde Google Sheets es posible modificar la información que tenemos en Memento. Ofrece la posibilidad de trabajar en equipo mediante Memento Cloud.

- Interplex es un programa de gestión de glosarios para iOS creado por un intérprete profesional (Peter Sand), especialmente diseñado para intérpretes de cabina que necesitan buscar términos con rapidez sin tener en cuenta acentos o palabras mal escritas.

\section{Dispositivos móviles y fuentes de información}

Una ventaja esencial que ofrecen los dispositivos móviles tanto para traductores como intérpretes es la posibilidad de tener acceso a Internet en cualquier momento y, por tanto, a la información que necesitemos. Esto hace posible el acceso a diccionarios o fuentes de información especializadas en línea en un momento dado si necesitamos cierta información. En algunos casos el acceso es restringido, no es gratuito. A modo de ejemplo destacamos algunos diccionarios en línea, en su mayoría gratuitos:

- Diccionario de términos médicos (DTM) de la Real Academia Nacional de Medicina (RANM), este diccionario no es gratuito pero es una fuente imprescindible para traductores e intérpretes médicos.

- Diccionario médico-biológico, histórico y etimológico de Ediciones Universidad de Salamanca, permite hacer búsquedas en español y en inglés de forma gratuita.

- Diccionario médico de la Universidad de Navarra, solo permite búsquedas en español, pero además de la definición del término ofrece artículos dónde encontrar más referencia de dicho término.

- Cercaterm del TermCat, permite búsquedas en muchos idiomas a partir del catalán y en muchas áreas. No es un diccionario al uso como explican en Sobre el Cercatem.

- Microsoft Language Portal, portal en abierto creado por Microsoft que da acceso a un glosario terminológico y a las cadenas localizadas de los productos localizados de Microsoft en todos los idiomas con los que trabaja Microsoft. Muy útil en la traducción técnica debido a que es terminología muy usada no solo por Microsoft.

- IATE, la base de datos terminológica de la UE que ofrece numerosas combinaciones de idiomas y varios campos de especialización. Esencial para traductores e intérpretes que trabajan en organismos relacionados con la UE.

- Glosbe, diccionario inglés-español, con otras combinaciones de idiomas posibles. Ofrecen el servicio de diccionario y además una memoria de traducción a partir de un corpus paralelo de textos origen y sus textos meta alineados. Los usuarios pueden añadir términos. 


\section{Dispositivos móviles y formación continua}

Una de las áreas que los dispositivos móviles nos han facilitado enormemente es el aprendizaje y la preparación para cada tarea que nos encargan. Obtener información para un proyecto de traducción o prepararse para sesiones de interpretación es una tarea que se ha simplificado porque no es necesario cargar con ningún tipo de material, podemos buscar información en cualquier momento, no estamos limitados a nuestro PC o a información física y, además, la tecnología móvil nos abre a un mundo de posibilidades.

La preparación para proyectos de traducción o bien para sesiones de interpretación es parte clave de nuestra profesión. En ciertos casos es posible que no se sepa específicamente en qué área hay que prepararse hasta poco antes de la sesión de interpretación por motivos de la política de confidencialidad de los hospitales, por ejemplo. En esos casos, la tableta o el móvil se convierte en el aliado del intérprete, la herramienta clave que hace posible el acceso a información esencial para informarse mínimamente, además de que permite consultar los glosarios personales del intérprete durante la sesión de interpretación.

Parte del trabajo del traductor e intérprete es la formación continua, estar actualizado sobre los temas en que suele trabajar, para ello nos podemos suscribir a los canales RSS de cualquier página que nos interese para recibir la información nueva. Una buena forma de gestionar esta información es con aplicaciones como Pocket para iOS, Feedly y Flipboard.

- Con el lector de noticias Pocket se pueden guardar artículos para leerlos más tarde desde cualquier sitio: Facebook, Twitter, Flipboard, Feedly, Safari, el correo electrónico, el PC. Una vez guardados, se pueden leer sin estar en línea.

- Feedly ofrece una versión gratuita y versiones para iOS, Android y otras plataformas. La información se puede compartir en las redes sociales, así como en OneNote 365 y Evernote Business. Su diseño es simple y se puede personalizar.

- $\quad$ Flipboard ofrece versiones para Android, iOS y Windows Phone. Permite ver las novedades de noticias, periódicos, redes sociales como Twitter, Facebook, así como organizar y compartir la información.

Los podcasts son otro tipo de fuente de información variada. Muchos medios de comunicación ofrecen podcasts, como BBC News. Con PocketCast, una aplicación tanto para iOS como para Android, podemos gestionar las suscripciones a podcasts y escucharlos cuando deseemos.

\section{Dispositivos móviles y gestión de la información}

Por otro lado, podemos contar con aplicaciones para la gestión de la información en cualquier dispositivo móvil. Entre las más recomendadas se encuentran:

- Evernote, una herramienta imprescindible y recomendada para organizar la información con versiones para iOS, Android y Windows Phone. Se pueden escribir notas, hacer listas, escanear, digitalizar distintos tipos de documentos físicos, sincronizar toda la información, guardar páginas web y organizarlas.

- Biscuit, es una aplicación que permite crear listas de palabras seleccionadas de artículos o cualquier página que nos interese. Ofrece también traducciones. Puede sincronizarse con Evernote para ofrecer más funciones, por ejemplo, a partir de la 
foto de una página, Biscuit y Evernote pueden generar una lista de palabras. Si son en inglés, Biscuit además las traduce.

- Scanner Pro es una herramienta que puede convertir cualquier foto de una página de un documento a PDF.

- Documents para iOS es una aplicación de gestión de archivos e información. Además de facilitar la organización de los numerosos tipos de archivos, permite ver, leer y anotar casi cualquier tipo de documento en el iPad o iPhone. También ofrece la posibilidad de subir archivos a Dropbox o Google Drive para compartir. Este tipo de aplicaciones son interesantes porque te permiten gestionar la información de forma similar a como se hace en los PC.

El almacenamiento en la nube hace posible la compartición de archivos entre dispositivos y PC con facilidad. Se puede acceder a la información que se copie en la nube en todo momento con nuestros dispositivos móviles. Además del almacenamiento en la nube gratuito (aunque limitado) que los distintos proveedores ofrecen (iCloud de Apple, Google Drive, Vodafone Cloud, etc.), podemos contar con otros sistemas de compartición de archivos como Dropbox o Box.

\section{Dispositivos móviles y comunicación}

No nos podemos olvidar de que los dispositivos móviles nos permiten acceso continuo a nuestro correo electrónico y, por tanto, es posible mantener el contacto con nuestros clientes incluso cuando no estamos delante de nuestro PC de trabajo. La angustia que puede sentir el traductor autónomo cuando espera cierta información o propuesta de trabajo de un cliente y siente que no puede moverse de su PC ha desaparecido. Podemos cuidar a nuestros clientes estemos donde estemos. Podemos atender nuestro trabajo desde un dispositivo móvil, estar en contacto con compañeros de profesión a través de las redes sociales o aplicaciones de chat. El traductor autónomo ya no está tan solo ni tan desconectado del mundo.

\section{Conclusión}

Los dispositivos móviles nos han abierto a un mundo de posibilidades en una profesión que depende en gran medida de la información y preparación que vayamos adquiriendo de forma continua y por nuestra cuenta. Nos han traído movilidad y nos ofrecen acceso a Internet desde cualquier sitio y en cualquier momento. Las posibilidades son infinitas, las aplicaciones que salen continuamente, algunas incluso diseñadas con la ayuda de profesionales de la traducción o interpretación, nos ofrecen nuevas posibilidades para mejorar nuestro trabajo, para hacerlo más eficaz. Podemos llevar nuestros diccionarios o glosarios sin hacer ningún esfuerzo físico, fácilmente en una tableta o incluso en un móvil, podemos usar las tabletas para tomar notas y consultar nuestras fuentes de referencia al mismo tiempo, podemos aprovechar el tiempo para mantenernos informados de los temas que nos interesan durante nuestros desplazamientos por trabajo, podemos llevar nuestros archivos de trabajo con nosotros en todo momento y mantenernos comunicados con nuestros clientes.

¿Cuánto tiempo falta para que se empiecen a desarrollar versiones móviles de los sistemas de memorias de traducción con integración de voz de forma que podamos ir dictando nuestras traducciones? Los dispositivos y las aplicaciones móviles están ayudando a derribar algunas barreras físicas que nos limitaban a un espacio de trabajo físico concreto. Pero todavía queda camino por recorrer, sobre todo en el área de los sistemas de memorias de traducción, no adaptados aún a plataformas móviles. 


\section{Referencias}

Dordas, Judith y Miguel Núñez. Dos palabras. Fecha de la última publicación: 27.09.11. Fecha de consulta: 09.10.16.

Drechsel, Alexander. The Tablet Interpreter. Fecha de la última publicación: 01.06.16. Fecha de consulta: 09.10.16.

Drechsel, Alexander y Josh Goldsmith. Tablet Interpreting: Tips, tools and applications to make the most of your tablet while interpreting. Fecha de publicación: 28.09.16. Fecha de consulta: 12.10.16.

Goldsmith, Josh. Consecutive 2.0. How to use your table for interpreting. IAPT 2015 Presentation, Bordeau. Fecha de publicación: 09.09.15. Fecha de consulta: 12.10.16.

González Álamo, Aida. Aventuras de una traductora-intérprete en Madrid. Fecha de la última publicación: 08.10.16. Fecha de consulta: 09.10.2016.

Hof, Michelle. The Interpreter Diaries. Fecha de la última publicación: 04.04.2016. Fecha de consulta: 09.10.16.

Rioja, Lourdes de. iPad Interpreter. Presentación de Alexander Drechsel. Fecha de publicación del vídeo: 18.07.11. Fecha de consulta: 09.10.2016.

Rioja, Lourdes de. The Tablet Interpreter's App Chart. Presentación de Alexander Drechsel. Fecha de publicación del vídeo: 21.10.14. Fecha de consulta: 09.10.16. 